\section{Estimating probability summation for binocular reaction time data}

\author{
RANDOLPH BLAKE, WILLIAM MARTENS, \\ and ANDREW GARRETT \\ Cresap Neuroscience Laboratory \\ Northwestern University \\ Evanston, Illinois 60201
}

and

DAVID WESTENDORF
University of Arkansas
Fayetteville, Arkansas 72701

On a host of visual tasks involving threshold measures, human observers benefit from using both eyes simultaneously, as compared to monocular viewing (Blake \& Fox, 1973). This improvement in two-eyed performance, known as binocular summation, could arise from some form of physiological interaction between the two eyes, an outcome referred to as neural summation. Alternatively, binocular summation could be due to the statistical fact that binocular viewing affords the observer two opportunities to see, in which case the enhancement in binocular performance would be attributable to probability summation.

In order to distinguish between these two alternative interpretations, neural vs. probability summation, it is necessary to estimate the level of performance expected from two independent chances to perceive and to compare this probability summation estimate with the performance level actually measured. In the case of threshold detection tasks, which comprise the bulk of the work on binocular summation, probability summation estimates may be derived either analytically or empirically. An analytical estimate involves using some formula or model of detectability (e.g., the integration model of signal detection theory) to derive a predicted value. The empirical approach involves measuring binocular performance under conditions which preclude any neural interaction, thus leaving probability summation as the only available mechanism. In the case of detection thresholds of various sorts, there is a fair amount of evidence to indicate which analytical formulations and which empirical control conditions provide valid estimates of probability summation (Eriksen \& Greenspon, 1968; Westendorf, Blake, \& Fox, 1972; Westendorf,

This work was supported by research grants from NIH (EY01596) and NSF (BNS7817948). R.B. holds a Career Development Award from NIH (EY00106). We thank L. E. Leguire for commenting on an earlier version of this note.
Langston, Chambers, \& Allegretti, 1978). Recently though, we have focused on simple reaction time (RT) as a gauge of binocular summation (Blake, Martens, \& DiGianfillipo, 1980; Westendorf, Blake, Sloane, \& Chambers, Note 1), and for this measure of visual performance there were no ready procedures for dealing with probability summation. Consequently, we have attempted to develop several strategies for estimating the level of binocular RT performance expected solely on the basis of probability. This note describes our progress along those lines.

To begin, it may not be so apparent that probability summation could contribute to binocular RT, since the visual targets themselves are typically detectable with a probability of 1.0 . Nonetheless, it is straightforward to conceptualize the RT task itself as involving a stochastic process, whereby the visual stimulus triggers a set of events (e.g., neural impulses) which can be characterized by some probability distribution. There are a number of RT models in which this represents a kernel assumption (e.g., McGill, 1963). From this conceptualization it immediately follows that multiple, simultaneous inputs in such a stochastic process will, on the average, yield shorter latencies. Just to illustrate, imagine thumbing through a full deck of shuffled playing cards at a steady pace and halting the first time you encounter a face card. If you repeat this procedure a number of times, always reshuffling and noting on each trial the amount of time taken to arrive at the first face card, you will generate a probability distribution of durations. Now imagine that both you and another person simultaneously perform this routine, each with your own shuffled decks, this time recording the length of time taken for either of you to encounter a face card. In the two-person case, it would take, on the average, a shorter duration to satisfy the face-card rule, simply on the basis of the laws of probability. And by the same token, two eyes performing the same task may produce shorter RTs based on the same statistical considerations. How may we go about estimating this level of performance?

\section{Graphic Solution}

One approach to the problem would be somehow to convert latencies directly to probability values and to use those values in concert with any of several available analytical formulae for predicting probability summation. In the case of simple RTs, however, there is no obvious rule for making this conversion. It is straightforward, however, to generate a cumulative frequency distribution of RTs and from such a distribution to determine the percentage of trials on which RT was equal to or less than some arbitrary value. Now, if we have two such RT distributions, one 
generated using just the left eye and the other just the right, we may derive two percentage values and use these to predict the percentage of trials on which binocular RTs should be equal to or less than that same arbitrary RT value, assuming independence of the two eyes. This predicted value may now be compared to the percentage value actually associated with the measured binocular RTs.

To illustrate the mechanics of this procedure, we have reanalyzed some $\mathrm{RT}$ data which was collected for other purposes (Blake et al., 1980), and representative results are shown in Figure 1 . The monocular and binocular RTs that comprise these cumulative distributions were collected for the same stimulus condition, as described in the figure caption. Once data are in this form, we select some RT value to generate proportions. In principle, any arbitrary value could be selected, but a very reasonable selection (especially when dealing with skewed distributions) is the median RT for the binocular condition, that is, the RT associated with the $50 \%$ point on the cumulative binocular function. We now determine by extrapolation the probabilities that the left-and right-eye RTs were less than this criterion. Those monoular probabilities can then be used to derive a probability summation estimate using the familiar theorem of independence:

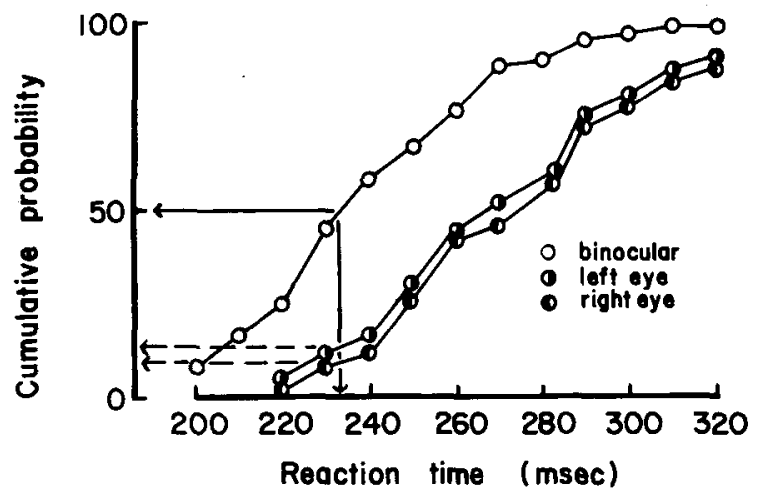

Figure 1. These cumulative frequency distributions show the probabilities of various reaction times (RT) for monocular and binocular viewing. These plots were generated using data collected in a simple $\mathrm{RT}$ paradigm (Blake et al., 1980). The $\mathrm{RT}$ stimulus was the abrupt appearance of a 1 cycle/deg vertical grating pattern of $10 \%$ contrast. Data were collected in blocks of 25 trials, with each block devoted to a particular viewing condition. The solid line indicates the $50 \%$ point on the binocular cumulative distribution; thus, during binocular testing, one-half of the measured RTs were equal to or less than the value denoted by the arrow along the abscissa. The dotted lines show the percentage of trials on which monocular viewing yielded RTs equal to or less than this criterion value. The resulting monocular percentages $(11 \%$ and $14 \%)$ can be used to compute a predicted binocular percentage. Employing Equation 1 (the classical theorem of independence), this predicted value is $231 / 2 \%$, which is significantly less than the obtained value of $50 \%$. This would constitute evidence in favor of neural, not probability, summation.

$$
P(B)=P(L)+P(R)-[P(L) \cdot P(R)],
$$

where $P(B), P(L)$, and $P(R)$ refer to binocular, left-eye, and right-eye probabilities, respectively. The difference between $P(B)$ and .50 (the actual value) can then be tested for statistical significance using any of several inferential techniques. Of course, evidence for genuine neural summation would be a value of $P(B)$ which was significantly smaller than $50 \%$. Statistical power could be achieved either by collecting a large number of monocular and binocular RTs or by collecting RTs in a randomized block design and applying the graphic analysis to each set of blocks. Incidentally, we (Blake et al., 1980) collected data in the latter fashion, and the results from this graphic solution overwhelmingly indicate that the improvement in binocular performance exceeds that predicted on the basis of probability summation.

It is worth noting that Equation 1 provides a liberal test of probability summation, in that it very likely overestimates the level of performance expected from two independent opportunities to detect (see Blake \& Fox, 1973, for a discussion of this issue). So rejection of the probability summation hypothesis based on this formula would constitute very strong evidence for neural summation.

\section{Solution by Simulation}

Another strategy which we have studied involves sampling twice from the distributions of monocular RTs, once from the left-eye distribution and once from the right-eye distribution. With each sample, we then take the briefer of the two values as the estimate of binocular RT, and from repeated samplings we generate a new RT distribution which represents an estimate of binocular performance expected from probability summation. This scheme treats the binocular condition as a race between two independent inputs, the winner being the first to arrive at the mechanism that triggers the RT.

We have simulated this model on a computer, again using the data of Blake et al. (1980), and the outcome is shown in Figure 2. The arithmetic mean of the probability summation sample is approximately $5 \%$ less than the mean of the monocular RTs, which merely confirms that two eyes should outperform a single eye just on the basis of statistical sampling considerations. Note, though, that the obtained distribution of binocular RTs differs from the simulated distribution, in the direction showing greater summation. This pattern of results would constitute evidence in favor of neural summation between the two eyes.

This particular simulation strategy makes no assumptions about the form of the monocular RT distributions, since it merely involves sampling from the obtained values themselves. It is assumed, however, that sampling proceeds in an unbiased fashion such 


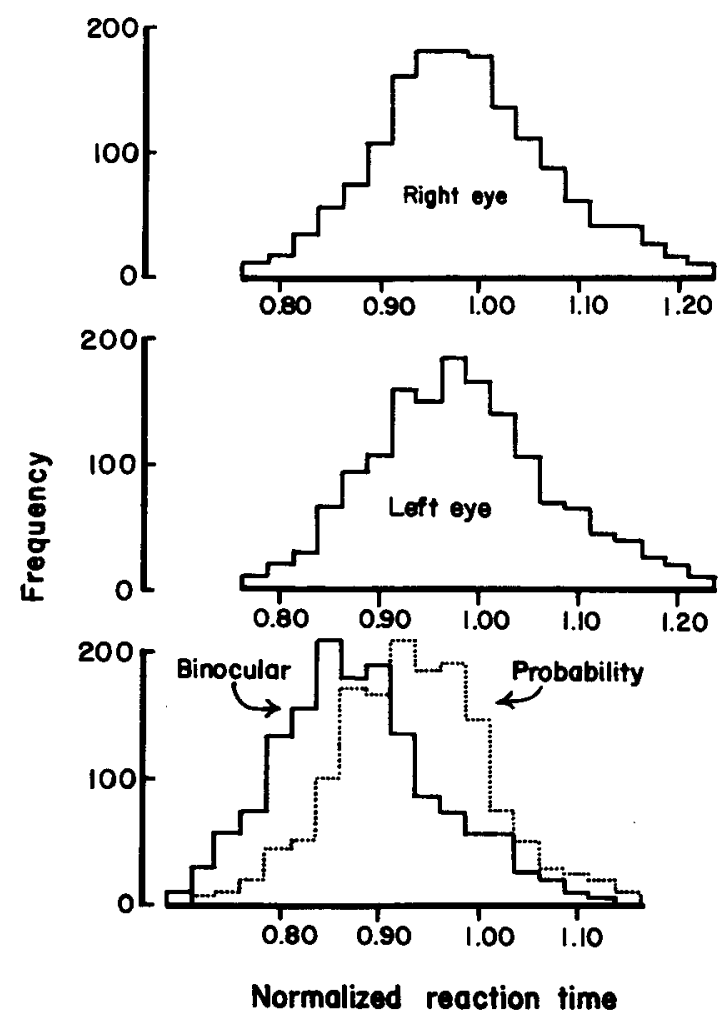

Figure 2. Frequency distributions of RT values measured using the right eye, the left eye, and both eyes (binocular). These data were taken from Blake et al. (1980); the RT values were normalized to allow us to pool data across subjects and conditions. The probability histogram (shown with dotted points) was generated by taking a random sample (with replacement) from the right-eye distribution and from the left-eye distribution and plotting the smaller of the two values for the probability condition.

that both monocular channels have equivalent independent access to the RT mechanism. If, however, this mechanism were envisioned as some type of periodic, serial scanning between the two eyes, the assumption of unbias might be violated. Likewise, the assumption of unbiased sampling could be violated if individuals exhibited marked eye dominance (Porac \& Coren, 1976). In either case, the sampling rule in the simulation would require revision, in order to weight the sample in favor of one eye. Any such revision would serve to reduce the predicted improvement in binocular RT owing to probability. So, again, the sampling model outlined here provides the most generous estimate of probability summation.

Going a step further, if we assume that the RT distributions for left-eye and right-eye conditions are equivalent and approximately normally distributed, the mean $\left(\mu_{B}\right)$ of the binocular RTs expected from two independent samples from the monocular distributions is given by

$$
\mu_{\mathrm{M}}=\mu_{\mathrm{M}}-(.56) \sigma_{\mathrm{M}}
$$

where $\mu_{\mathrm{M}}$ and $\sigma_{\mathrm{M}}$ refer to the mean and standard deviation of the monocular RTs; this formulation follows from statistical sampling theory (e.g., David, 1970). An obvious advantage to this estimation procedure is that it obviates the necessity of generating via sampling an entire RT distribution. Rather only two values, the mean and standard deviation of one set of monocular RTs, are needed.

\section{Empirical Estimates}

Finally, there is a third general approach to the problem of probability summation, whereby binocular performance is assessed under conditions that rule out neural interaction between the two eyes. In the case of threshold tasks, such conditions have included stimulation of noncorresponding retinal areas (Westendorf \& Fox, 1977), separation of the two monocular inputs over time (Matin, 1962; Westendorf, Langston, Chambers, \& Allegretti, 1978), and stimulation with dissimilar monocular targets (Westendorf, Blake, \& Fox, 1972; Westendorf \& Fox, 1974). In other work (Blake et al., 1980), we have measured binocular RTs using dissimilar stimulation and using stimulation of noncorresponding areas of the two eyes. In both instances, the improvement in binocular performance was on the order of $5 \%$, relative to monocular performance. While very similar to the improvement predicted by Equation 2, this level of improvement consistently underestimates binocular performance with identical stimulation of corresponding areas.

\section{Conclusions}

Estimating probability summation for RT data, while not quite so straightforward as in the case of detection thresholds, may be possible using any of several different strategies. All of the strategies outlined here involve some set of assumptions, but none of these assumptions seems grossly unwarranted. Of course, the appropriateness of any one of these strategies must be evaluated on the basis of some validating procedure, such as an empirical control condition. In addition, it may prove worthwhile to collect monocular and binocular RT data under conditions involving more complicated decision rules which encourage tradeoffs between speed and accuracy. In this way, it may be possible to separate the contributions of genuine neural summation between the two eyes from those associated with variations in decision, or criterion, factors. Finally, it may prove useful to employ these various strategies for estimating probability summation in other RT paradigms where multiple channels are thought to be activated (e.g., stimulation involving two or more separate retinal regions). And, in addition to RT, they may have applicability to other suprathreshold visual tasks, such as magnitude estimation. 


\section{REFERENCE NOTE}

1. Westendorf, D., Blake, R., Sloane, M., \& Chambers, D. Binocular summation during suppression. Manuscript in preparation.

\section{REFERENCES}

Blake, R., \& Fox, R. The psychophysical inquiry into binocular summation. Perception \& Psychophysics, 1973, 14, 161-185.

Blake, R., Martens, W., \& Digianfillipo, A. Reaction time as a measure of binocular interaction in human vision. Investigative Ophthalmology and Visual Science, in press.

David, J. Order statistics. New York: Wiley, 1970.

Eriksen, C. W., \& Greenspon, T. S. Binocular summation over time in the perception of form at brief durations. Journal of Experimental Psychology, 1968, 76, 331-336.

MAtin, L. Binocular summation at the absolute threshold for peripheral vision. Journal of the Optical Society of America, $1962,52,1276-1286$.
MCGiLL, W. J. Stochastic latency mechanisms. In R. D. Luce, R. R. Bush, \& E. Galanter (Eds.), Handbook of mathematical psychology (Vol. 1). New York: Wiley, 1963.

Porac, C., \& Coren, S. The dominant eye. Psychological Bulletin, 1976, 83, 880-897.

Westendorf, D. H., Blake, R. R., \& Fox, R. Binocular summation of equal-energy flashes of unequal duration. Perception \& Psychophysics, 1972, 12, 445-448.

Westendorf, D., \& Fox, R. Binocular detection of positive and negative flashes. Perception \& Psychophysics, 1974, 15, 61-68.

WestendoRF, D., \& Fox, R. Binocular detection of disparate light flashes. Vision Research, 1977, 17, 697-703.

Westendorf, D., Langston, A., Chambers, D., \& Allegretti, C. Binocular detection by normal and stereoblind observers. Perception \& Psychophysics, 1978, 24, 209-213.

(Received for publication February 6, 1980; Accepted February 12, 1980.) 